—Sie sollte regelmäßig (je nach Praxisgröße täglich bis wöchentlich) auf einem externen Speichermedium vorgenommen werden. Hierzu eignen sich etwa gut RAID-Systeme (Redundant Array of Independent Disks). Dahinter verbirgt sich ein Verbund unabhängiger Festplatten in einem Gehäuse. Die Daten werden beim Speichern gleichzeitig auf mehreren Festplatten abgelegt. Das senkt das Risiko, dass sie in irgendeiner Form nicht auslesbar sind. In kleinen Praxen kann aber auch eine normale USB-Festplatte für die Datensicherung genutzt werden.

_Die Datensicherung sollte immer getrennt vom Rechnersystem der Praxis aufbewahrt und vor allem nicht dauerhaft an dieses angeschlossen werden. Denn: „Viele Verschlüsselungs- trojaner können auch Daten auf externen und Netzwerklaufwerken unbrauchbar machen", mahnt das BSI.

- Außerdem sollte das Praxisteam anhand einiger ausgewählter Dateien prüfen, ob sich die gesicherten Dateien tatsächlich wiederherstellen lassen und die Datensicherung funktioniert.

\section{Am besten das ganze Programm ...}

Zusätzlich benötigen Praxen Schutzmechanismen, die Eindringlinge von vornherein abwehren. Dazu zählen in jedem Fall eine aktivierte, aktuelle Firewall (die meisten Internet-Router haben bereits eine integrierte Firewall), ein aktuell gehaltener Antivirenscanner sowie ein Betriebssystem und ein Internetbrowser, die durch regelmäßige Updates dem neuesten Sicherheitsstandard ent- sprechen. Wichtig ist laut BSI darüber hinaus eine gesunde Portion Misstrauen gegenüber unbekannten E-Mail-Absendern, etwa mit Rechnungsanhang. Gleiche Vorsicht gelte bei E-Mail-Faxen. Beim Surfen im Internet hilft es, wenn der Virenscanner sichere und unsichere Websites symbolisch kennzeichnet. Praxen müssen außerdem die Schweigepflicht auch für die Patientendaten auf den Rechnern beachten. Es gilt daher, die Systeme - falls der Nachweis doch einmal strafrechtlich nötig ist - nach bestem Wissen durch den aktuellen Stand der Technik zu schützen.

Der Internist geht heute sogar noch einen Schritt weiter: „Der Mailrechner wird vom Netz und vom Server abgehängt; nur dort lokal geprüfte Mails werden weiter geleitet.“ Rebekka Höhl

\title{
Die private Suchmaschine für Gesundheitsdaten
}

\section{Das E-Health-Gesetz ermöglicht, dass künftig viele verschiedene Gesund- heitsdaten über die Telematikinfrastruktur fließen. Private Suchmaschinen könnten Ärzten hier helfen, die für sie entscheidenden Daten herauszufiltern.}

$\mathrm{D}$ iagnosen und Befunde, Verordnungen, Arztberichte, aber auch von den Patienten über Fitness-Tracker oder Apps selbst erhobene Gesundheitsdaten sollen Medizinern künftig über die sichere, gemeinsame Datenautobahn - die Telematikinfrastruktur (TI) - zur Verfügung stehen. Abgelegt wird alles auf verschiedensten Servern. Doch selbst, wenn die elektronische Patientenakte kommt: Für den einzelnen Arzt wird es wichtig sein, die für ihn entscheidenden Patientendaten herauszufiltern. Die österreichischen Nachbarn, deren Gesundheitskarte ELGA bereits seit 2015 in Betrieb ist und derzeit in zwei Bundesländern angewandt wird, sind hier schon weiter: Dort wurde im Pilotversuch mit einem Klinikverbund die Suchmaschine der Mindbreeze GmbH getestet. Wie Dr. Adolf Sonnleitner, bei Mindbreeze für Kunden im Bereich Gesundheitswesen zuständig, erläutert, funktioniert die Such-Applikation im Prinzip ähnlich wie Google. Mit dem Unterschied: Die Anwender erhalten eine Server-Box, auf der sich die Such-Applikation befindet, sie müssen also keine Software auf die Rechner spielen. Die Applikation lasse sich dennoch nach eigenen Kriterien konfigurieren. Dabei greife sie nicht nur auf interne Daten zu. Angebunden an eine zentrale Datenautobahn, wie die TI in Deutschland, könnten auch externe Datenbanken beziehungsweise Server gefiltert werden. Damit sollen die Ärzte der Flut an Daten Herr werden.

In Österreich werden Befunde derzeit laut Sonnleitner auf zwölf verschiedenen Domänen abgelegt. „Ein Arzt kann sich etwa alle Befunde $\mathrm{zu}$ einem Patienten, nennen wir ihn Herrn Schmidt, heraussuchen lassen“, sagt Sonnleitner. Er könne aber auch noch detaillierter etwa nach Fachbegriffen oder ausschließlich Infos, die sich um die Diabetes-Erkrankung von Herrn Schmidt drehen, suchen. Sonnleitner: „Der Augenarzt wird sich nicht durch hunderte Befunde arbeiten wollen, die ihn größtenteils nicht betreffen." Neben der Volltextsuche kann deshalb nach Fachbereichen, Arzneimitteln, nur Arztbriefen, ICD-Codes, Laborwerten und ähnlichem gefiltert werden. Der Datenschutz wird gewahrt, indem der Such-Applikation die für die Praxis oder Gesundheitsdatenautobahn geltenden Berechtigungsverfahren vorgeschaltet werden. „Als Zugangsberechtigung zur E-Health-Plattform bleiben die Gesundheitskarte und der Arztausweis erhalten“, stellt Sonnleitner klar. Der Arzt muss sich also als Behandler ausweisen, die Daten werden ihm dann auch nur für eine begrenzte Zeit bereitgestellt.

\section{Ein Cockpit für Patienten}

Ob irgendwann eine Anbindung der Such-Applikation von Mindbreeze an die TI möglich ist, ist noch nicht klar. Das Gespräch mit der gematik habe man bislang noch nicht gesucht. Sonnleitner sagt auch ehrlich, dass sich die Lösung für Praxen erst dann lohnt, wenn sie in zentralen Strukturen arbeiten und hunderte Befunde durchsuchen müssen. „Denn dann müssen die Systeme so nutzbar sein, dass der Arzt nicht 10 bis 20 Minuten mit der Datensichtung beschäftigt ist."

Im Prinzip werde mit der privaten Suchmaschine eine Art Wissensdatenbank aufgebaut, so umschreibt es Mindbreeze-Geschäftsführer Daniel Fallmann. Zukünftig sollen die Ärzte so dann auch eine Art Cockpit für ihre Patienten bekommen.

RebekkaHöh 